\title{
A Unified Switch Loss Model and Design Consideration for Multilevel Boost PFC With GaN Devices
}

\author{
Jiawen WU, Yu QI, Faheem MUHAMMAD, and Xinke WU
}

\begin{abstract}
Recently, multilevel converters with gallium nitride (GaN) devices have shown marvelous advantages for power factor correction (PFC) conversion to meet the increasingly higher efficiency and power density requirements. In the traditional design process for the multilevel PFC converter, it is necessary to separately optimize the devices of the corresponding breakdown voltage under different level number, which causes difficulty to the overall optimization of the entire system. In this paper, a unified minimum loss model for GaN switches regardless of voltage levels is proposed to optimize the efficiency based on device's new figure-of-merit (NFoM) $\left(N F o M=C_{\mathrm{OSS}(\mathrm{ER})} R_{\mathrm{DS}(\text { on })}\right)$. With the help of this unified minimum loss model, it simplifies the efficiency optimizing methodology according to the NFoMs of GaN devices for multilevel PFC converter. According to the methodology, a $2 \mathrm{~kW}$ cascaded H-bridge (CHB) PFC prototype is constructed to verify the design methodology, achieving over $99 \%$ efficiency with power density over $1000 \mathrm{~W} / \mathrm{in}^{3}$.
\end{abstract}

Index Terms - Design methodology, gallium nitride(GaN), high efficiency, multilevel, new figure-of-merit (NFoM).

\section{INTRODUCTION}

$\mathrm{F}^{\mathrm{o}}$ OR electric vehicle chargers, internet data-center power systems and cellphone base station power supply, a compact and efficient ac/dc converter is required [1], [2].As a result, among these applications, high efficiency and high power density are the two most important motivations [3], [4], presenting increasing challenges, in the past couple of years.

Traditionally, the most popular power factor correction (PFC) topology is a diode bridge rectifier cascaded with a boost converter. However, due to the high conduction loss in rectifier diodes, the PFC efficiency is less than $98 \%$. For the sake of eliminating the diode bridge loss, the totem-pole bridgeless PFC has been widely used in low power ac/dc applications [5], [6]. Although the efficiency can be improved to $99 \%$, the power density is still limited owing to the large size of boost

Manuscript received February 3, 2021; revised April 13, 2021; accepted June 29, 2021. Date of publication December 30, 2021; date of current version December 29,2021 . This work was supported in part by the National Natural Science Foundation of China under Grant 51877191 \& 52177197 and in part by Zhejiang Natural Science Outstanding Young Scholar Foundation under Grant LR18070001. (Corresponding author: Xinke Wu.)

J. Wu is with Inovance (Suzhou) Co., Ltd., Suzhou, China.

Y. Qi is with Silergy Semiconductor Technology (Hangzhou) Co., Ltd., Hangzhou, China.

F. Muhammad and X. WU are with Zhejiang University and Hangzhou Global Scientific and Technological Innovation Center, Zhejiang University, Hangzhou, China.

Digital Object Identifier 10.24295/CPSSTPEA.2021.00032 inductor. The efficiency of the PFC stage is improved to $99.3 \%$ with cascaded H-bridge (CHB) multilevel topology [4], [7], whose switching frequency is around $20 \mathrm{kHz}$. However, the power density is sacrificed by the low switching frequency. Thanks to the better properties of gallium nitride $(\mathrm{GaN})$ devices and soft switching utilization, the switching frequency was successfully pushed to one megahertz and the power density of the totem-pole PFC power stage achieved around $250 \mathrm{~W} / \mathrm{in}^{3}$ [8]. However, the inductor still occupies the largest part of volume in PFC stage except for the bulky dc bus capacitors, even though the switching frequency has reached megahertz.

To achieve higher power density, priority should be given to reducing the size of the inductor [9]. To keep the same current ripple requirements, the minimized volt-second of the inductor in the multilevel topology can lower the inductance significantly because the inductance value is squarely reciprocal to level number [10]. With the reduced volt-second and inductance, the inductor volume will be reduced by 95\% when 7 levels are used [11]. Without the bulky volume occupied by the inductor, the system power density can be further improved. Recently, with flying capacitor multilevel topology, the power density of the PFC power stage is improved to over $500 \mathrm{~W} / \mathrm{in}^{3}$ [12], [13].

Apart from the power density, the efficiency of multilevel converter is also an important concern, which has not been analyzed systematically in previous work and analytical loss model is absent. In the traditional loss model, the figure of merit (FoM) is utilized to calculate the losses of devices and get the minimum loss of switches in conventional pulse width modulation (PWM) converters, where the voltage stresses are determined according to their individual application requirements [14]-[16]. However, the voltage stress of devices is unknown for the multilevel converter until the voltage level is determined. Thus, the optimal efficiency points with different voltage levels have to be calculated separately by traversing all possible devices with different breakdown voltage to obtain the optimal voltage level and achieve the highest efficiency. As a result, it is not only complex but also time-consuming to optimize the efficiency for multilevel converter with the traditional loss model.

In order to simplify the design process for multilevel converter, a unified loss model of device for multilevel converter has been presented briefly in [17]. In this paper, more detailed derivations and experimental verifications are provided. The unified minimal analytical loss model of switches for the basic multilevel topology is with a different 


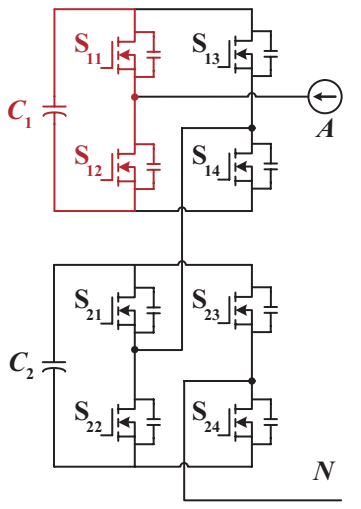

(a)

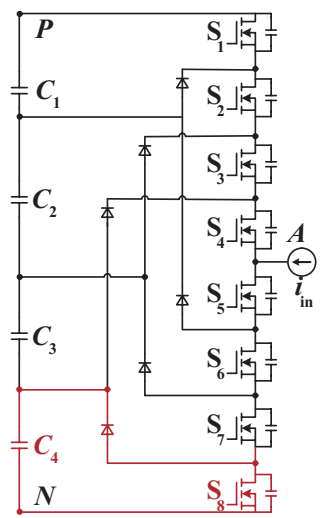

(c)

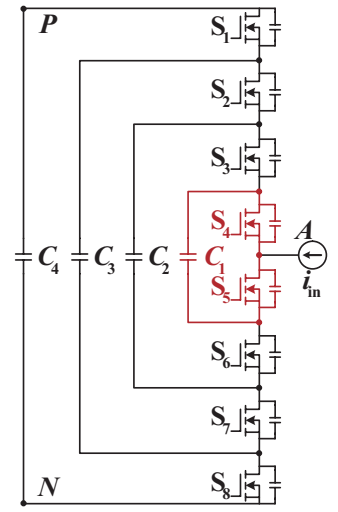

(b)

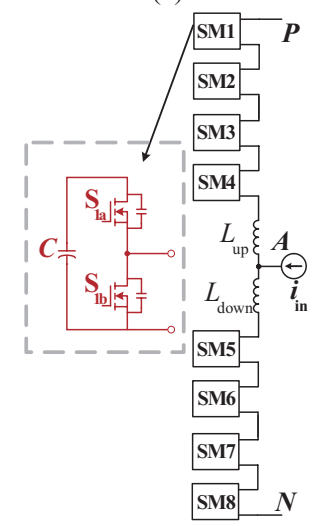

(d)
Fig. 1. Typical multilevel (5 level) topologies: (a) CHB, (b) FC, (c) NPC and (d) MMC.

figure of merit (new figure of merit-NFoM) [18], [19]. Based on this unified loss model, a design methodology aiming at achieving the highest power density under the same efficiency requirement is presented. According to the proposed design methodology, the optimal voltage level and the switching frequency can be easily obtained without complex calculation process and the analysis has been verified with experiments.

\section{NFoM Based Minimal Loss Model for GAN Switches}

\section{A. Conventional Loss Model for GaN Devices}

Fig.1 shows typical multilevel candidate topologies including cascade $\mathrm{h}$ bridge (CHB), flying capacitor (FC), neutral point clamping (NPC) and modular multilevel converter (MMC) [20], [21] for PFC applications. All candidates are composed of the same basic structure, which is shown in Fig. 2 (a). And the basic unit is used to calculate the loss because the total loss on switching device can be easily derived by multiplying the unit loss by the unit number and this derivation is suitable for all multilevel topologies. Here, other loss, including loss on bus capacitor, loss on inductor and loss on filter, are not included in the following discussion for the simplicity and generality of the calculation model, because they are topology-related. Since this basic unit is the same for all topologies mentioned in Fig.1, this abstraction process helps to unify and simplify the loss

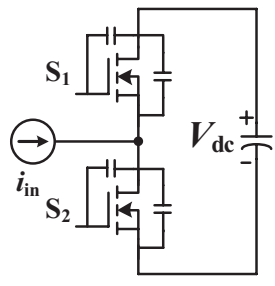

(a)

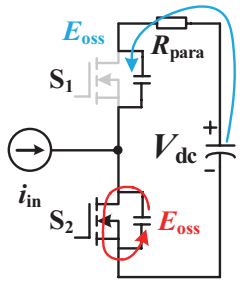

(b)
Fig. 2. Basic half bridge cell of multilevel boost PFC topologies: (a) Cell structure. (b) Capacitive loss during hard switching on.

calculation. This basic half bridge structure includes a current source, a half-bridge circuit and a dc voltage source. For CHB, NPC and MMC the voltage is the dc voltage of each cell, while for $\mathrm{FC}$ that is the equivalent dc source, which is the difference between two flying capacitors.

Power device loss in one basic half bridge cell includes conduction loss, capacitive loss, switching loss and driving loss. The reverse recovery loss of body diode is negligible in $\mathrm{GaN}$ device, which will not be included in the analysis.

Firstly, the conduction loss can be calculated by (1).

$$
P_{\text {con }}=k_{\text {temp }} k_{\mathrm{dyn}} I_{\text {in }}^{2} R_{\mathrm{DS}(\mathrm{on})}
$$

Where $k_{\text {temp }}$ and $k_{\text {dyn }}$ are the junction temperature coefficient and the dynamic resistance coefficient, respectively [22].

When it comes to the switching loss, it includes turn-on loss, turn-off loss and capacitive loss. For the low voltage GaN devices with hard switching, the piecewise linear analytical model is used here[15], [16].

$$
\left\{\begin{array}{l}
P_{\mathrm{on}}=\frac{V_{\mathrm{dc}} I_{\mathrm{in}} R_{\mathrm{g}(\mathrm{on})}}{2\left(V_{\mathrm{dri}}-V_{\mathrm{pl}}\right)}\left(Q_{\mathrm{gs} 2}+Q_{\mathrm{gd}}\right) f_{\mathrm{s}} \\
P_{\mathrm{off}}=\frac{V_{\mathrm{dc}} I_{\mathrm{in}} R_{\mathrm{g}(\mathrm{off})}}{2 V_{\mathrm{pl}}}\left(Q_{\mathrm{gs} 2}+Q_{\mathrm{gd}}\right) f_{\mathrm{s}}
\end{array}\right.
$$

where $Q_{\mathrm{gs} 2}$ is the gate charge that charges the gate capacitance from the threshold voltage to the miller platform voltage; $Q_{\mathrm{gd}}$ is the gate to drain charge, $V_{\mathrm{dri}}$ is the gate driving voltage; $V_{\mathrm{pl}}$ is the miller platform voltage and $R_{\mathrm{g}(\mathrm{on})} / R_{\mathrm{g}(\mathrm{off})}$ is the gate charging resistor for turning on and off respectively.

As shown in Fig. 2 (a), $S_{1}$ is considered as a rectifier when the input current flows into the middle point of half bridge, so its turn-on loss is ignored here due to zero voltage switching (ZVS). So, the switching loss of the basic half bridge cell includes turn-on loss of $\mathrm{S}_{2}$ and turn-off losses of $\mathrm{S}_{1}$ and $\mathrm{S}_{2}$, which are combined into (3) according to (2).

$$
P_{\mathrm{sw}}=V_{\mathrm{dc}} I_{\mathrm{in}} k_{\mathrm{g}}\left(Q_{\mathrm{gs} 2}+Q_{\mathrm{gd}}\right) f_{\mathrm{s}}
$$

where $k_{\mathrm{g}}=R_{\mathrm{g}(\mathrm{on})} / 2\left(V_{\mathrm{dri}}-V_{\mathrm{pl}}\right)+R_{\mathrm{g}(\mathrm{off})} / V_{\mathrm{pl}}$.

For hard switching conditions, not only the turn-on loss, but also the capacitive loss of each turn-on process should be considered, as shown in Fig. 2 (b). $E_{\text {oss }}$ is defined as the energy 
stored by the parasitic capacitor of each switch. When $\mathrm{S}_{2}$ is turned on, $2 E_{\text {oss }}$ will be dissipated, half of which is depleted by the channel of $\mathrm{S}_{2}$, and the rest is dissipated by the parasitic loop impedance. Therefore, the capacitive loss of the basic half bridge cell can be derived by (4).

$$
P_{\text {oss }}=2 E_{\text {oss }} f_{\mathrm{s}}=Q_{\text {oss }} V_{\mathrm{dc}} f_{\mathrm{s}}
$$

where $Q_{\text {oss }}$ is the output charge, which can be obtained from the datasheet of the manufacturer.

The device loss calculation can be simplified by using figure of merit. (2) and (3) are rewritten as (5) [16].

$$
\left\{\begin{array}{l}
P_{\mathrm{sw}}=k_{\mathrm{g}} V_{\mathrm{dc}} I_{\mathrm{in}} F o M_{\mathrm{HS}} f_{\mathrm{s}} / R_{\mathrm{DS}(\mathrm{on})} \\
P_{\mathrm{oss}}=V_{\mathrm{dc}} f_{\mathrm{s}} F o M_{\mathrm{oss}} / R_{\mathrm{DS}(\mathrm{on})}
\end{array}\right.
$$

where $F o M_{\mathrm{HS}}=\left(Q_{\mathrm{gs} 2}+Q_{\mathrm{gd})}\right) R_{\mathrm{DS}(\text { on })} ; F o M_{\mathrm{oss}}=Q_{\mathrm{oss}} R_{\mathrm{DS}(\text { on })}$.

For $\mathrm{GaN}$ device with switching frequency below $100 \mathrm{kHz}$, the driving loss is relatively small, which can be ignored here. Combining (1) and (5), the total device loss of the bridge in Fig. 2 is described in (6) [16].

$$
\begin{array}{r}
P_{\mathrm{GaN}}=k_{\mathrm{temp}} k_{\mathrm{dyn}} I_{\mathrm{in}}^{2} R_{\mathrm{DS}(\mathrm{on})}+V_{\mathrm{dc}}\left(I_{\mathrm{in}} k_{\mathrm{g}} F o M_{\mathrm{HS}}+F o M_{\text {oss }}\right) f_{\mathrm{s}} / R_{\mathrm{DS}(\mathrm{on})}(6) \\
A \times R_{\mathrm{DS}(\mathrm{on})}+B / R_{\mathrm{DS}(\mathrm{on})} \geqslant 2 \sqrt{A B}
\end{array}
$$

According to (6) and basic inequality (7), the minimal device loss model can be deduced as (8).

$$
\begin{gathered}
P_{\mathrm{GaN}_{-} \text {min }}=2 I_{\mathrm{in}} \sqrt{V_{\mathrm{dc}} k_{\mathrm{temp}} k_{\mathrm{dyn}}\left(k_{\mathrm{g}} I_{\text {in }} F o M_{\mathrm{HS}}+F o M_{\mathrm{oss}}\right) f_{\mathrm{s}}} \\
R_{\mathrm{DS}(\mathrm{on})}=\frac{1}{I_{\mathrm{in}}} \sqrt{\frac{V_{\mathrm{dc}}\left(I_{\mathrm{in}} k_{\mathrm{g}} F o M_{\mathrm{HS}}+F o M_{\text {oss }}\right) f_{\mathrm{s}}}{k_{\mathrm{temp}} k_{\mathrm{dyn}}}}
\end{gathered}
$$

Here, $F o M_{\mathrm{HS}}$ and $F o M_{\mathrm{OSS}}$ in the loss model vary with the voltage stress of the switching devices. In conventional PWM converter, the voltage stress is determined according to its individual specification. So $F o M_{\mathrm{HS}}$ and $F o M_{\mathrm{Oss}}$ are determined. Then, the minimal loss model in (8) can be utilized to derive the optimal efficiency. But, for the multilevel converter, the voltage stress is related to the voltage level. The highest efficiency cannot be projected with (8) directly before the voltage stress is determined. Meanwhile, the voltage level will affect the optimal efficiency point of the multilevel converter. Therefore, it is hard to select an optimal voltage level according to the FoM based minimal loss mode in (8). Due to the unknown relationship between the voltage level quantity and the minimal loss, an iterative method among all possible amounts of voltage level has to be applied. Thus, to obtain the minimal loss among all possible devices and voltage levels, different voltage stresses for kinds of series of devices are calculated based repeatedly.

\section{B. Unified Minimal Loss Model Based on NFoM of GaN De- vices in Multilevel Converters}

A minimal loss model based on NFoM [18] of devices, has

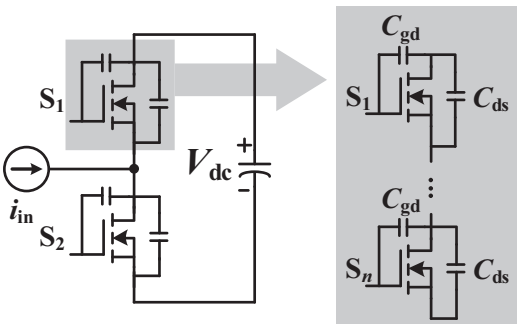

Fig. 3. Low voltage devices in series instead of high voltage devices.

been proposed for resonant DCX [19], which eliminates the voltage stress of GaN HEMTs in the loss model. Therefore, the series connected low voltage devices can be evaluated and compared to high voltage devices by NFoM. It is expected that for multilevel PWM converter the minimal loss model could be a guideline of determining the voltage level with the highest efficiency.

For the loss analysis, the cell circuits with series connection are equivalent to low voltage devices connected in series, as shown in Fig. 3.

For each low voltage device, the minimal loss (8) can be used, where the dc voltages across the switches are identically assumed to be $V_{\mathrm{dc}} / N$. Since the $N$ number of devices have been connected in series, the total-minimal loss of $N$ seriesconnected devices are obtained by (10).

$$
P_{\text {GaN_min }}=2 I_{\text {in }} \sqrt{N V_{\text {dc }} k_{\text {temp }} k_{\text {dyn }}\left(k_{\mathrm{g}} I_{\text {in }} F o M_{\text {HS }}+F o M_{\text {oss }}\right) f_{\mathrm{s}}}
$$

For CHB, this minimum loss should be multiplied by 2 because each cell consists of two half bridges. Besides, (10) shows that the minimum loss is related to both $N$ and FoM, so the optimal $N$ and the minimum loss cannot be obtained directly. In order to solve this problem, (10) needs to be further simplified.

Firstly, $F o M_{\text {oss }}=\left(C_{\mathrm{OSS}(\mathrm{ER})} V_{\mathrm{dc}}\right) R_{\mathrm{DS}(\mathrm{On})} / N\left(C_{\mathrm{OSS}(\mathrm{ER})}\right.$ is the energy equivalent capacitance). (10) can be reorganized in (11).

$$
P_{\text {GaN_min }}=2 I_{\text {in }} \sqrt{N V_{\text {dc }} k_{\text {temp }} k_{\text {dyn }}\left(k_{\mathrm{g}} I_{\text {in }} F O M_{\mathrm{HS}}+\frac{V_{\text {dc }}}{N} N F O M\right) f_{\mathrm{s}}}
$$

where $N F o M=C_{\mathrm{OSS}(\mathrm{ER})} R_{\mathrm{DS}(\text { on) }}$.

Secondly, in the expression of $F o M_{\mathrm{HS}}, Q_{\mathrm{gd}}$ is related to the voltage stress and $Q_{\mathrm{gs} 2}$ is related to gate voltage, the ratio between $Q_{\mathrm{gd}}$ and $Q_{\mathrm{gs} 2}$ varies with voltage stress, which is shown in Fig. 4(a) with regard to $100 \mathrm{~V} 4 \mathrm{~m} \Omega \mathrm{GaN}$ device [23]. By curve fitting, the coefficient can be obtained from the dashed line in Fig. 4(a). For the sake of simplicity, all devices use the same $k_{\mathrm{g} 2 \mathrm{~d}}$ described in Fig. 4(a), which results in less than $10 \%$ deviation of switching loss for today's low voltage rating devices, as shown in Fig. 4(b).

Then, $F o M_{\mathrm{HS}}$ is simplified in (12).

$$
F o M_{\mathrm{HS}}=\frac{V_{\mathrm{dc}}}{N}\left(1+k_{\mathrm{g} 2 \mathrm{~d}}\right) N F o M_{\mathrm{sw}}
$$

Finally, by substituting (12) into (11), a uniform minimal 


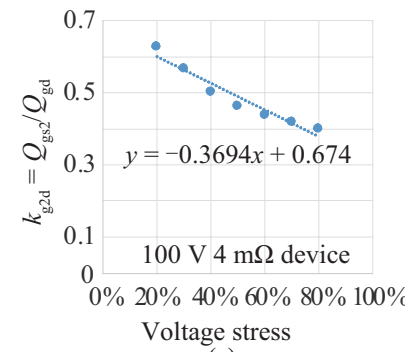

(a)

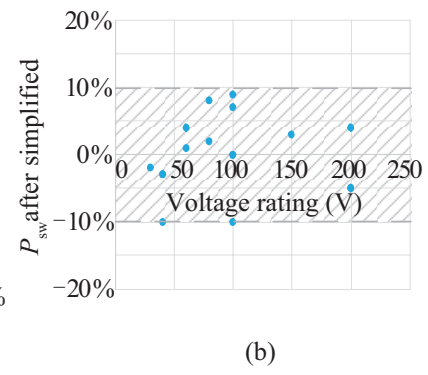

(b)
Fig. 4. Simplification. (a) Coefficient under different voltage stress. (b) Switching loss difference after simplified.

loss model for multilevel converter with GaN devices can be derived by (13).

$$
P_{\text {GaN_min }}=2 V_{\text {dc }} I_{\text {in }} \sqrt{k_{\text {temp }} k_{\text {dyn }}\left(k_{\text {sw }} I_{\text {in }} N F O M_{\text {sw }}+N F o M\right) f_{\mathrm{s}}}(13)
$$

where $N F o M_{\mathrm{sw}}=C_{\mathrm{GD}(\mathrm{TR})} R_{\mathrm{DS}(\mathrm{on})} ; N F o M=C_{\mathrm{OSS}(\mathrm{ER})} R_{\mathrm{DS}(\mathrm{on})} ; k_{\mathrm{sw}}=$ $k_{\mathrm{g}}\left(1+k_{\mathrm{g} 2 \mathrm{~d}}\right)$.

Following the same logic, the optimal $R_{\mathrm{DS}(\text { on })}$ in (9) can also be simplified to (14).

$$
R_{\mathrm{DS}(\text { on })}=\frac{V_{\mathrm{dc}}}{I_{\mathrm{in}}} \sqrt{\frac{\left(I_{\mathrm{in}} k_{\mathrm{sw}} N F o M_{\mathrm{sw}}+N F o M\right) f_{\mathrm{s}}}{k_{\mathrm{temp}} k_{\mathrm{dyn}}}}
$$

The simplified uniform loss model in (13) shows that the capacitance and $R_{\mathrm{DS}(\mathrm{on})}$ of the single devices determine the minimum loss jointly. This conclusion can be briefly explained from a physical point of view. Firstly, the loss of seriesconnected circuit is the same as devices connected in series as shown in Fig. 3, where capacitances and on resistances are all connected in series. Secondly, the equivalent on-state resistance is proportional to $N$ while the equivalent capacitance is inversely proportional. Thus, the product of them keeps the same. Finally, $N F O M$ and $N F O M_{\text {sw }}$ for $N$ series-connected devices keep the same as that of a single device, which means that the loss of entire system is irrelevant with $N$.

In a nutshell, the improvement of this uniform loss model is that the minimal loss models of both high voltage devices and low voltage devices are unified without considering voltage stress because the minimal loss is merely dependent on the individual device's NFOM.

\section{Density OptimizATION Method FOR Multilevel PFC CONVERTER}

In order to demonstrate the guideline of the proposed uniform loss model in selecting the voltage level and achieving the optimal efficiency of multilevel PFC converter, the CHB topology with unipolar modulation scheme is presented. Here, only the front stage is considered for simplicity, and when the latter isolated dc-dc is taken into consideration, present works [24], [25] can be referred to for further optimization.

The topology for $N_{\text {cells }}=n$ (number of cells) is shown in Fig. 5(a). With the phase-shift unipolar modulation, each device is switched at the same frequency, which will reduce the volt-

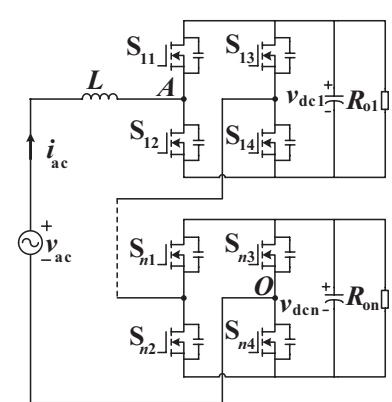

(a)

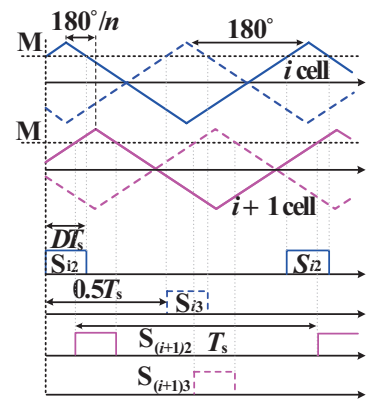

(b)
Fig. 5. CHB (a) topology for $N_{\text {cells }}=n$ and (b) modulation scheme.

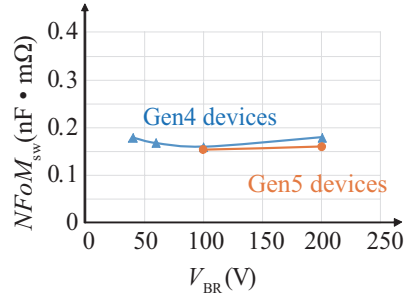

(a)

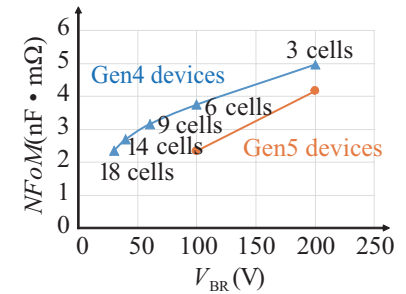

(b)
Fig. 6. GaN device characteristics (a) $N F o M_{\mathrm{sw}}$ and (b) NFoM.

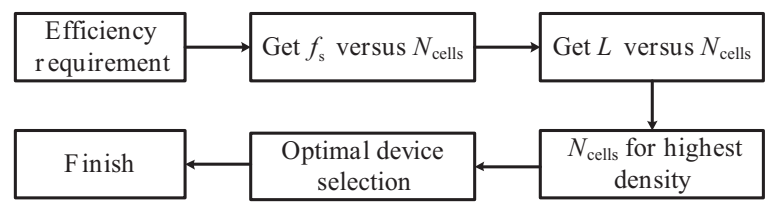

Fig. 7. Procedure of efficiency optimization by selecting voltage level.

second across the input inductor. Each cell circuit consists of two bridges, whose carriers take on 180 degrees phase shift. The phase shift among cell circuits are 180/ $n$ degrees, as shown in Fig. 5 (b).

According to the unified minimal loss model, device loss is only related to the $N F O M$ and $N F O M_{\text {sw }}$ of a single device. For today's technology, the $R_{\mathrm{DS}(\text { on })}$ of $\mathrm{GaN}$ device is far from the limit [26], [27]. Through curve fitting, $R_{\mathrm{DS}(\text { on) }}$ is in proportional to $V_{B R}{ }^{0.8}$. Meanwhile $C_{\mathrm{gd}}$ is almost inversely proportional to $V_{B R}{ }^{0.8}[28]$, which results in an almost constant $N F O M_{\text {sw }}$, as Fig. 6(a) illustrated. Meanwhile, $N F O M$ increases as the breakdown voltage of device increases as shown in Fig. 6(b) [22], [23], [26], which means that devices with lower breakdown voltage are able to achieve lower loss of the entire system.

Fig. 7 shows a simple flow chart to illustrate the entire selection process. In the first step, the efficiency requirement should be established. For example, $99 \%$ efficiency is required under $3 / 4$ load and $380 \mathrm{~V}$ dc bus.

Secondly, to meet the requirement of $99 \%$ efficiency, $0.5 \%$ $\mathrm{GaN}$ devices loss is usually permitted. Under the same expected loss limitation, the relationship between $f_{s}$ and NFoM for each cell number is derived from (13). The modified equation is derived in (15), where $\lambda$ is the permitted loss percentage. 


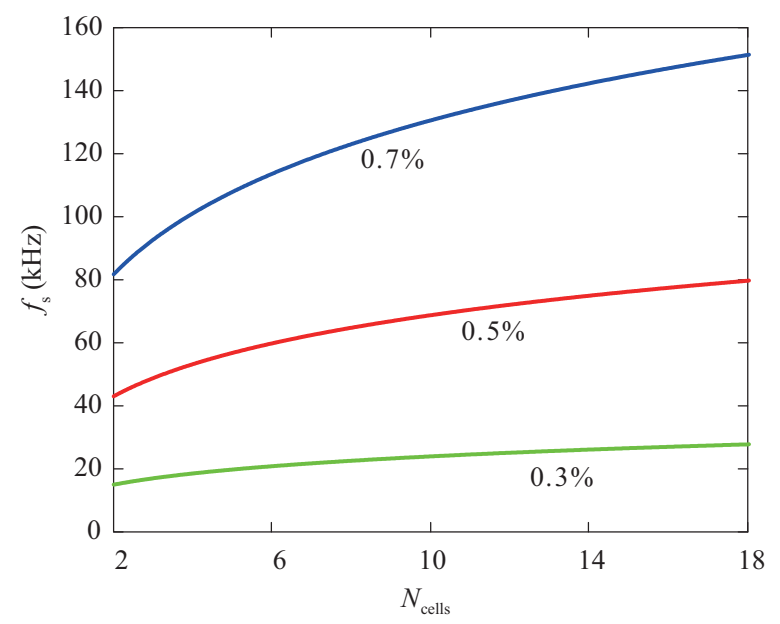

Fig. 8. The maximal frequency under different loss limitations $(0.3 \%, 0.5 \%$, $0.7 \%$ ) of devices.

$$
f_{\mathrm{s}} \leqslant \frac{\lambda^{2} P_{\mathrm{o}}^{2}}{16 V_{\mathrm{dc}}^{2} I_{\mathrm{in}}^{2} k_{\mathrm{temp}} k_{\mathrm{dyn}}\left(k_{\mathrm{sw}} I_{\mathrm{in}} N F O M_{\mathrm{sw}}+N F O M\right)}
$$

According to the characteristics of the fourth-generation commercial devices, the relationship between NFOM and the total cell number $N_{\text {cells }}$ can be fitted, and then the maximal switching frequency corresponding to different cell numbers is obtained. The results are depicted in Fig. 8. Here, the limitations of device loss of $0.3 \%, 0.5 \%$ and $0.7 \%$ are given as examples. If higher loss is allowed, the maximal frequency could be higher. Under the same loss limitation, the implementation of more cells leads to higher acceptable switching frequency.

Thirdly, the relationship between inductor and $N_{\text {cells }}$ should be established. Although more cells adoption shrinks inductor size, large amounts of devices and size of PCB traces area will reduce the power density. In contrary, less cells results in larger inductor size, which may also sacrifice the power density. Therefore, a suitable cell number and the inductor volume should be compromised according to their relationship.

The volt-second of the inductor during the whole line operation is calculated by (16) and plotted in Fig. 9, where only $\pi / 2$ is provided because of its symmetry operation during half line cycle. This formula is a piecewise function, where different natural number $k$ means an antithetic section.

$$
\text { Volt }- \text { second }=\frac{V_{\mathrm{dc}}\left(1-D_{\mathrm{eq}}\right) D_{\mathrm{eq}}}{N_{\text {cells }}} \frac{1}{2 N_{\text {cells }} f_{\mathrm{s}}}
$$

where $D_{\text {eq }}=D-0.5(k-1) / N_{\text {cells }} ; D=\left(1-v_{\text {ac }} / V_{\text {dc }}\right) / 2$ and $0.5(k-1) /$ $N_{\text {cells }} \leqslant D \leqslant 0.5 k / N_{\text {cells }}$ for $k=1,2, \ldots, 2 N_{\text {cells }}$.

As shown in Fig. 9, within each section, volt-second increases from zero first and then decreases as the line angle increases. When $D_{\text {eq }}$ is equal to 0.5 , the volt-second of the inductor reaches its peak value. With a given switching frequency, the maximal volt-second will decrease squarely as the number of cells increases. And the inductance can be designed according to its maximum volt-second in (17), where

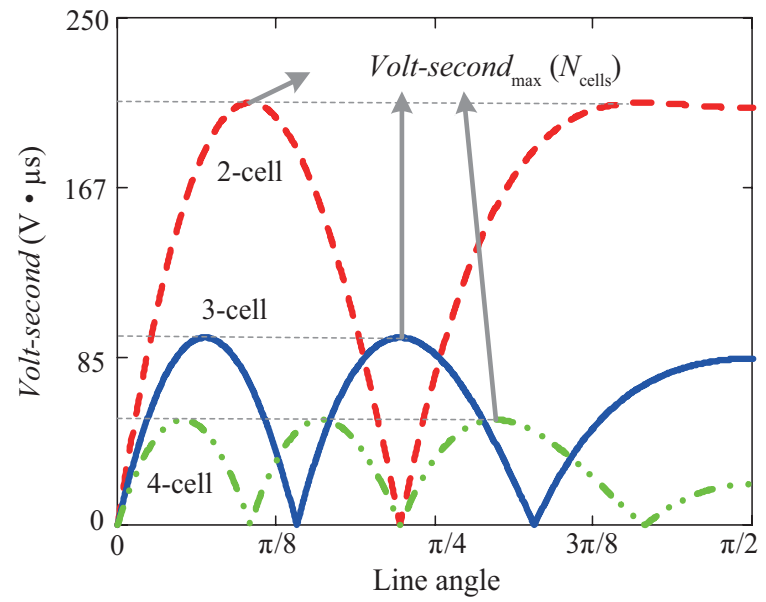

Fig. 9. The volt-second of the inductance with different cells at $60 \mathrm{kHz}$ switching frequency and $220 \mathrm{~V}$ ac input voltage.

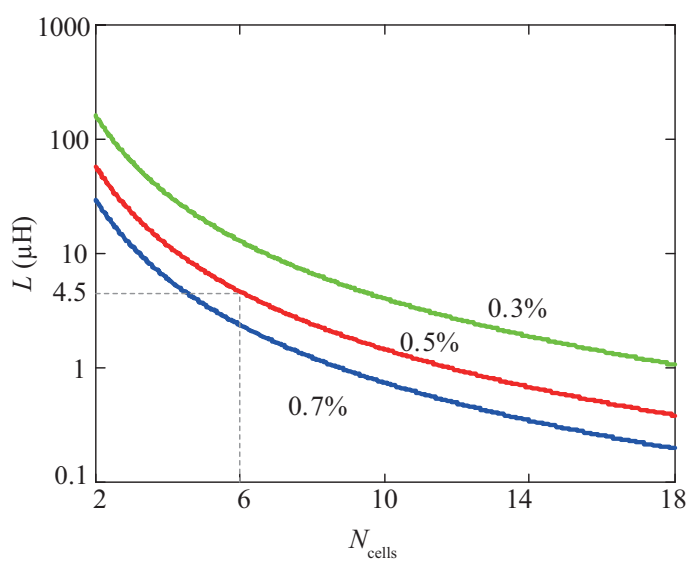

Fig. 10. The inductance under different loss limitations of devices.

$\Delta i_{\text {pk-pk }}$ is the maximal peak to peak current ripple and is chosen around $20 \%$ of the maximal peak to peak ac current in this work.

$$
L=\frac{\text { Volt }- \text { second }_{\mathrm{max}}\left(N_{\mathrm{cells}}\right)}{\Delta i_{\mathrm{pk}-\mathrm{pk}}}=\frac{V_{\mathrm{dc}}}{8 N_{\text {cells }}^{2} f_{\mathrm{s}} \Delta i_{\mathrm{pk}-\mathrm{pk}}}
$$

According to (17), the inductance for different cell number is plotted in Fig. 10. Here, limitations of $0.3 \%, 0.5 \%$ and $0.7 \%$ of device loss are given as the examples.

In order to optimize the whole system's power density, cell number is optimized firstly here. Since the volume of circuit cells and inductor determine the entire volume. Their relationship with $N_{\text {cells }}$ is plotted in Fig. 11. On one hand, the inductor size decreases as the cell number increases as plotted with the circle blue points shown in Fig. 11. On the other hand, the volume of main circuit, which includes GaN devices, driver and power isolator, is plotted as the triangle green points. The chip ISOW7840 used in each cell to provide the functions of digital signal isolation and power supply takes up the largest volume in a cell and it is the same for all level number. Meanwhile, though the size of GaN MOSFET changes with the level number, it is comparatively small. Thus, the relationship 


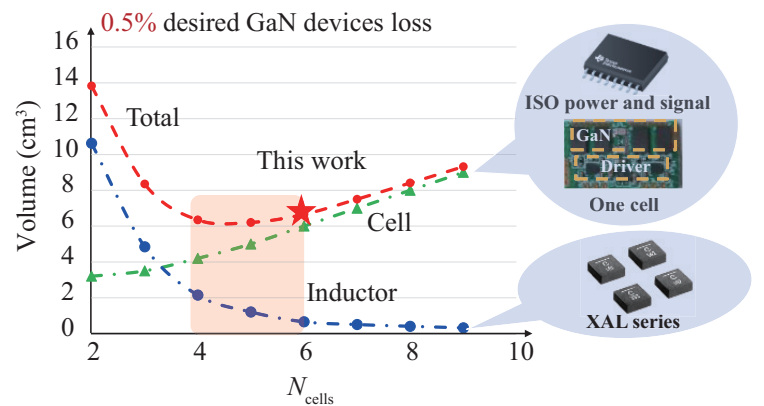

Fig. 11. Volume trade-off between the cell circuits and the inductor.

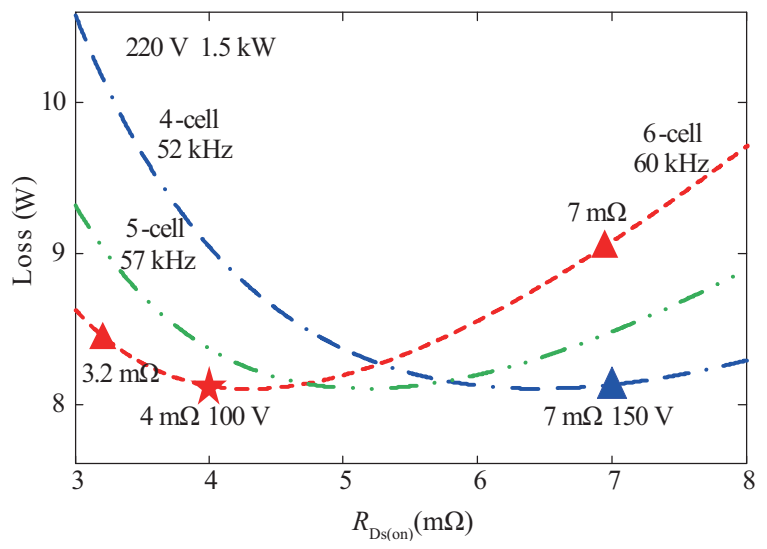

Fig. 12. Device $R_{\mathrm{DS}(\text { on) }}$ selection under different cells (4, 5 and 6).

between the volume of the main circuit and level number is approximately linear as shown in Fig. 11. In a whole, the red points are the total volume of the inductor and all cells, which shows that the volumes of cell number 4,5 and 6 differ little, among which the cell number is optimal to be chosen.

Fig. 12 shows the relationship of loss and $R_{\mathrm{DS}(\mathrm{n})}$ of commercial GaN devices, where the blue, green, and red curves are drawn with the design of 4-cell, 5-cell, and 6-cell, respectively. The best device selections at $4 \mathrm{~m} \Omega$ with $100 \mathrm{~V}$ and $7 \mathrm{~m} \Omega$ with $150 \mathrm{~V}$ are suitably preferred. However, commercial halfbridge non-isolated GaN driver is designed to withstand only up to $100 \mathrm{~V}$. Thus, the 6-cell structure with device of 100 $\mathrm{V}$ breakdown is chosen in this paper and the $R_{\mathrm{DS}(\mathrm{on})}$ is $4 \mathrm{~m} \Omega$. Finally, the inductance value can be selected according to Fig. 10. In 6-cell system, if $0.5 \%$ loss limitation is chosen, the switching frequency is chosen as $60 \mathrm{kHz}$, which is not high while the equivalent switching frequency is $720 \mathrm{kHz}$ due to the phase-shift control. Besides, the multilevel structure also helps to reduce the voltage step on inductor. So, the inductance of the inductor is fairly small, which is only $4.5 \mu \mathrm{H}$ in our prototype.

\section{COMPARISON With Si Devices}

Si device can also be used in this application but performs not as good as GaN. In order to compare $\mathrm{Si}$ and $\mathrm{GaN}$ fairly, $\mathrm{Si}$ and $\mathrm{GaN}$ with the same breakdown voltage and the same $R_{\mathrm{DSon}}$ are chosen. For Si, BSC040N10NS5 is chosen and EPC2032 is chosen for $\mathrm{GaN}$, whose breakdown voltage are both $100 \mathrm{~V}$, and the $R_{\mathrm{DSon}}$ are

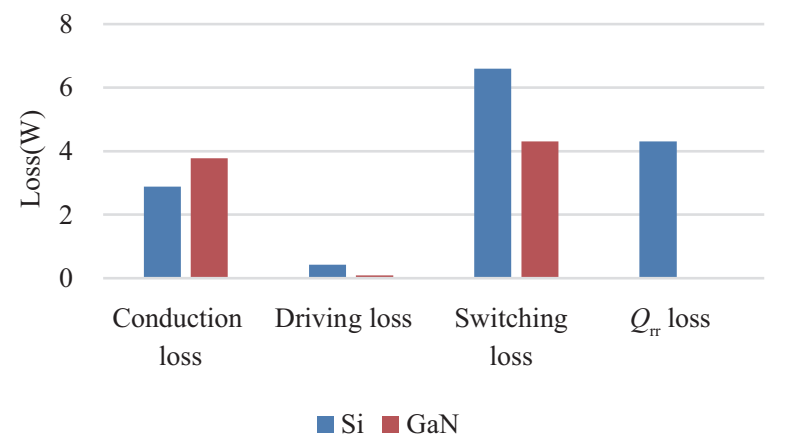

Fig. 13. Loss comparison between $\mathrm{GaN}$ and $\mathrm{Si}$.

both $4 \mathrm{mR}$. The following three aspects are discussed.

\section{A. Loss Comparison}

The loss comparison is conducted at $75 \%$ of the full load. When $\mathrm{Si}$ is used, the loss of the devices is $14 \mathrm{~W}$ for 6 cells $\mathrm{CHB}$, and when $\mathrm{GaN}$ is used, the loss at the same condition is only $8 \mathrm{~W}$. So, the loss of $\mathrm{Si}$ is about two times of $\mathrm{GaN}$ and the loss breakdown comparison is shown as Fig. 13. The conduction loss of $\mathrm{GaN}$ is a little larger than $\mathrm{Si}$ due to its dynamic resistor phenomenon and apart from that, the loss of $\mathrm{GaN}$ is all smaller than Si. Especially, GaN does not have $Q_{\mathrm{rr}}$ loss, which accounts for a large portion for Si device. In addition, the $Q_{\mathrm{g}}$ of $\mathrm{GaN}$ is $12 \mathrm{nC}$ and the $Q_{\mathrm{g}}$ of $\mathrm{Si}$ is $58 \mathrm{nC}$. Therefore, the driving loss of $\mathrm{GaN}$ is far smaller than that of $\mathrm{Si}$, which will make the design of the driving circuit easier and facilitate future integration.

\section{B. Price Comparison}

Prices of both devices are searched on Digit-Key, and the price for BSC040N10NS5 is $\$ 3.45000$ per chip and $\$ 6.83000$ for EPC2032. Indeed, at present, GaN does not have the price advantage over Si because GaN has not yet been widely produced. However, after mass production in the future, the cost of GaN may be close to that of Si.

\section{Size Comparison}

Besides, the size of devices is also important. The available $\mathrm{Si}$ devices are usually larger than the GaN device that we chose. For example, the size of BSC040N10NS5 is $5.5 \mathrm{~mm} \times$ $6.35 \mathrm{~mm} \approx 35 \mathrm{~mm}^{2}$ per chip, while the size of EPC2032 is only $4.63 \mathrm{~mm} \times 2.63 \mathrm{~mm} \approx 12 \mathrm{~mm}^{2}$. Thus, the size of Si device is approximately three times of that of GaN device and using GaN devices helps to shrink the system's volume.

In a nutshell, though Si device can be used in this topology, it is inferior to $\mathrm{GaN}$ in terms of efficiency and volume. Besides, massive production and improvement in the future will help to reduce the price along with enhancing GaN's performance.

\section{EXPERIMENTAL VERIFICATIONS}

A single-phase PFC prototype with $2 \mathrm{~kW}$ rated power is built and tested, whose prototype is shown Fig. 14. Since the 


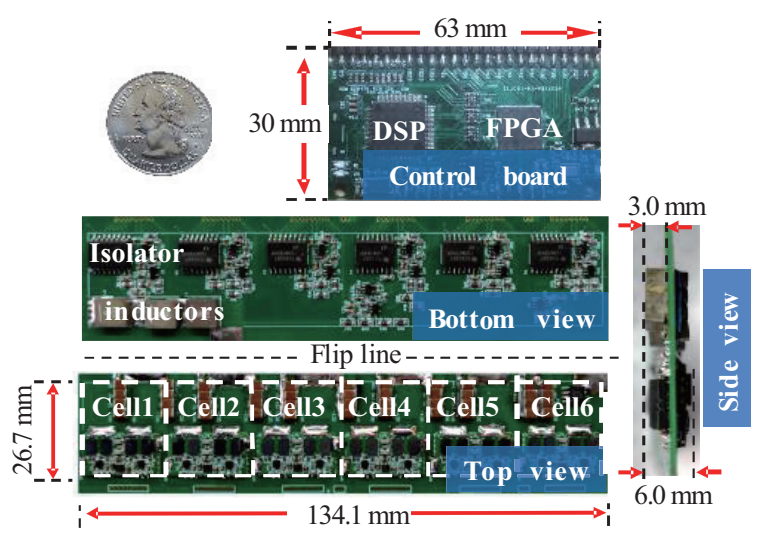

Fig. 14. Photograph of the prototype.

TABLE I

Key Parameters of the Prototype

\begin{tabular}{ll}
\hline \hline Description & Parameters \\
\hline Switches & $24 \times$ EPC2032 \\
Inductors $L$ & $3 \times$ XAL7030-1.5 $\mu \mathrm{H}$ \\
Power and signal isolator & $6 \times$ ISOW7840 \\
Switching frequency & $60 \mathrm{kHz}$ \\
Filter inductance $L_{\mathrm{dm}}$ & $8 \mu \mathrm{H}$ \\
Filter capacitance $C_{1}$ & $620 \mathrm{nF}$ \\
DC voltage & $60 \mathrm{~V} \times 6$ \\
Rated power & $2 \mathrm{~kW}$ for $220 V_{\text {ac }}$ \\
Density (with control) & $1050 \mathrm{~W} / \mathrm{in}^{3}$ \\
\hline
\end{tabular}

low profile of the overall system is beneficial for the reduction the system's volume, XAL7030, whose height is only $3.1 \mathrm{~mm}$, is selected and three of them are used in series to minimize the height of the converter. For a better illustration, a coin of the quarter is used to visualize the prototype size. The power density of the power stage achieves over $1000 \mathrm{~W} / \mathrm{in}^{3}$ with a digital control board. The key parameters of the prototype are listed in Table I.

Fig. 15 shows the simplified schematic for the prototype where resistive load is used to verify the design. For CHB topology, a cascaded second isolated $\mathrm{dc} / \mathrm{dc}$ stage is usually added to avoid the circulating current among the multiple low voltage dc buses in typical ac/dc applications [29], [30]. In this case, dc voltage balance loop can be added to control each dc voltage, which has been discussed in reference [31]-[34].

Fig. 16 illustrates the key waveforms of input current and inductor current, input voltages of ac side and switch side $V_{\mathrm{AO}}$, at $110 V_{\text {ac }}$ input. With the phase shifted modulation strategy, the voltage step of $V_{\mathrm{AO}}$ is around $60 \mathrm{~V}$. The peak voltage of 110 $V_{\text {ac }}$ is $155 \mathrm{~V}$, which results in only 7 voltage levels in the phase voltage $V_{\mathrm{AO}}$.

Fig. 17 gives the key waveforms at $220 V_{\text {ac }}$ input. Maximum 13 levels can be observed in the phase voltage $V_{\mathrm{AO}}$. The grid current is well-fitted in phase in both cases when the $V_{\mathrm{ac}}$ input voltage is $110 \mathrm{~V}$ and $220 \mathrm{~V}$. The dc bus voltages of cells no. 5 and no. 6 are almost the same. Hence, the system dc balance is achieved, as shown in Fig. 18.

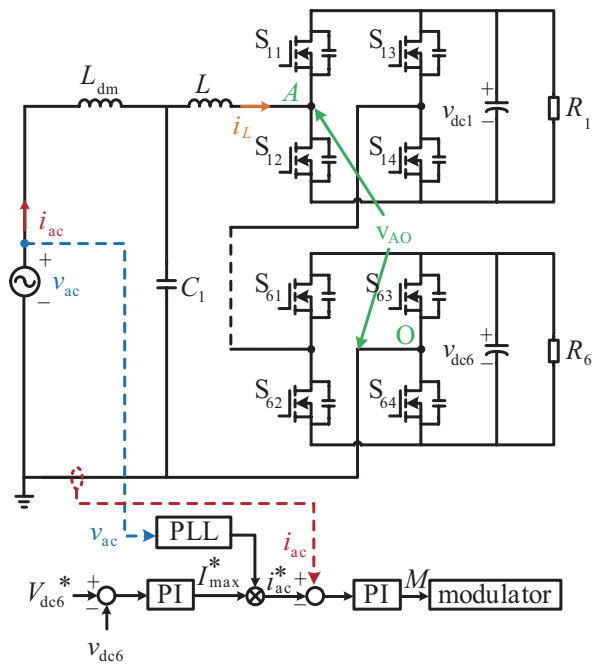

Fig. 15. Experimental system structure.

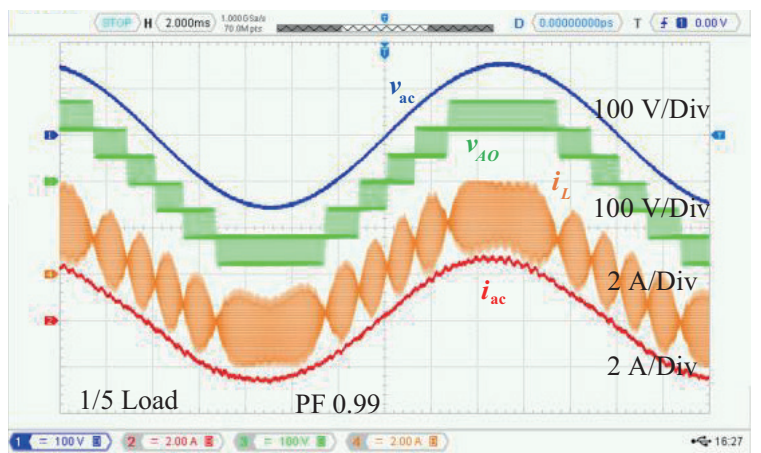

(a)

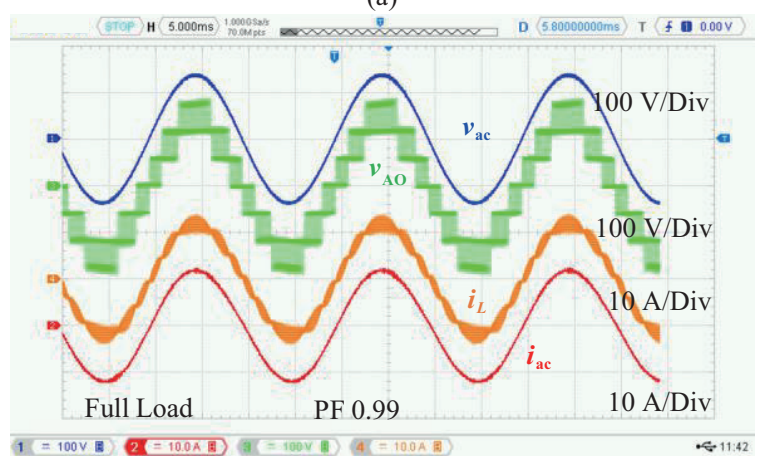

(b)

Fig. 16. Key voltage and current waveforms at $110 V_{\text {ac }}$ input. (a) $20 \%$ load and (b) full load.

The current total harmonic distortion (THD) achieves around $1 \%$ under full load condition and less than $5 \%$ under $10 \%$ of full load condition at both $110 V_{\text {ac }}$ and $220 V_{\text {ac }}$ input voltages. The graphical representation is shown in Fig. 19.

Efficiencies are shown in Fig. 20 for different input voltage. The dashed lines represent the mathematically-calculated efficiency result, where the inductor loss is based on the simulation tool given by the manufacturer, bus cap loss is calculated with the parameters of EKZN800ELL182MM40S and GaN HEMTs loss are based on the loss model in Part II. In the model, $k_{\text {temp }}$ is 1.3 for $50{ }^{\circ} \mathrm{C}$ temperature rise according to 


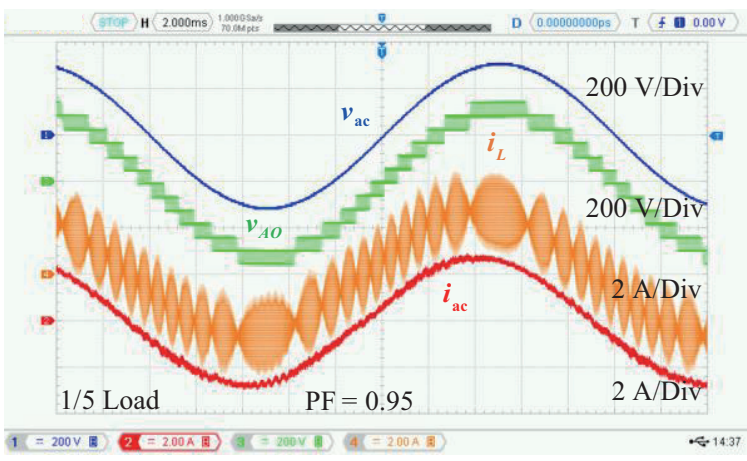

(a)

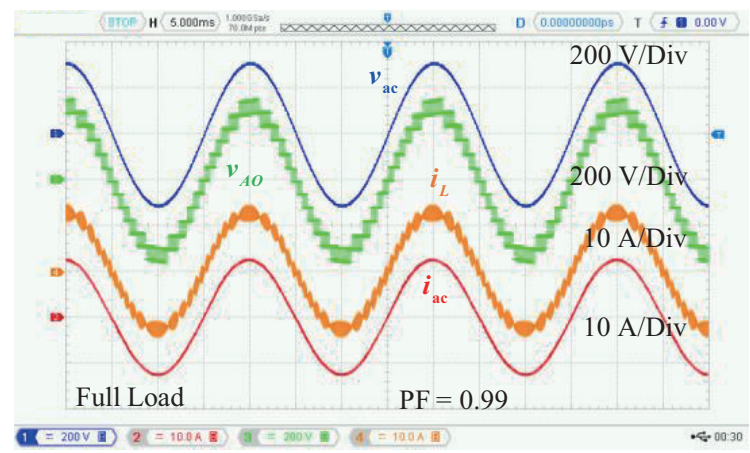

(b)

Fig. 17. Key voltage and current waveforms at $220 V_{\text {ac }}$ input. (a) $20 \%$ load and (b) full load.

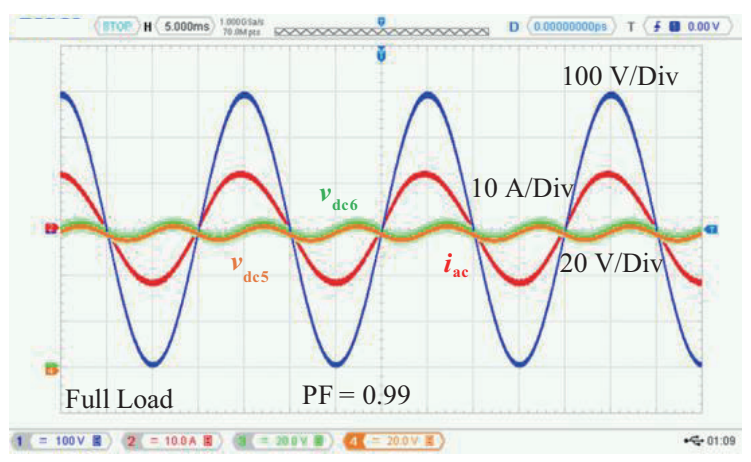

Fig. 18. Key dc voltage waveforms at $220 V_{\text {ac }}$ input.

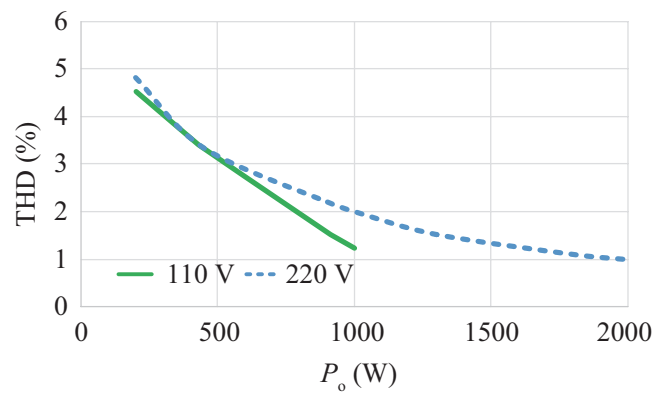

Fig. 19. THD of the input current.

the datasheet and $k_{\mathrm{dyn}}$ is 1.3 for $60 \mathrm{kHz}$ operation [22]. Besides, $R_{\mathrm{g}(\mathrm{on})}$ is $3 \Omega$ and $R_{\mathrm{g}(\mathrm{off})}$ is $1 \Omega$, representing the resistance of

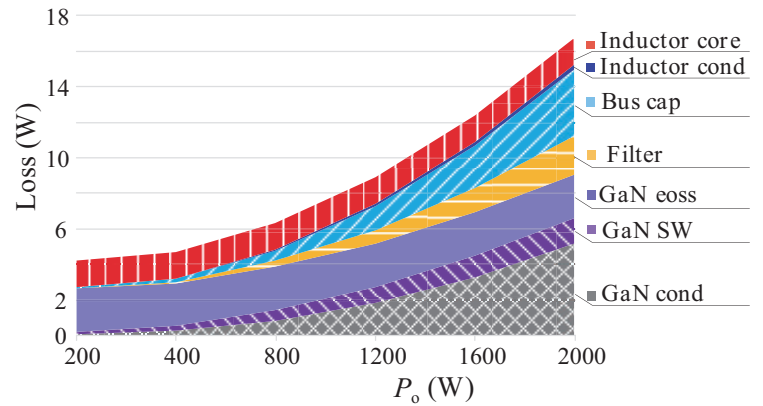

(a)

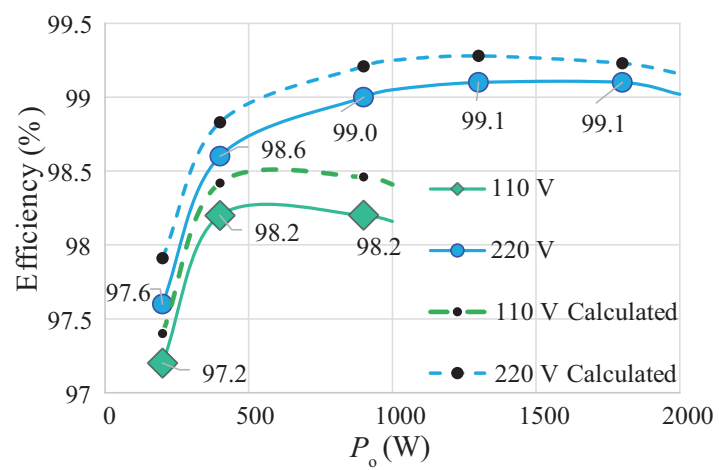

(b)

Fig. 20. Loss breakdown and efficiency comparison with different input voltage and loads. (a) Loss breakdown for $220 V_{\text {ac }}$ input. (b) Efficiency comparison between calculation and experimental results.
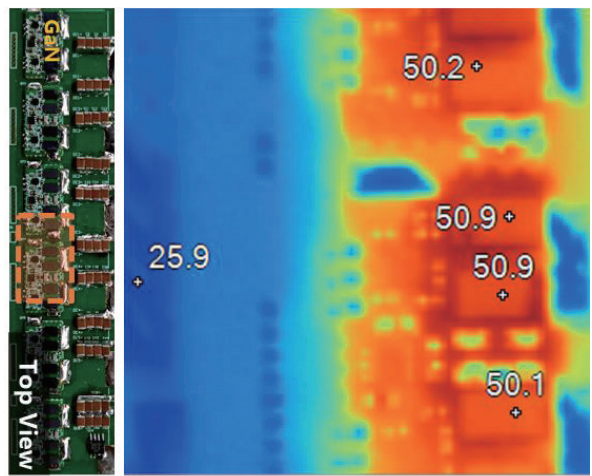

Fig. 21. Thermal graph of $75 \%$ load experiment.

the driver LMG1205 and the GaN HEMT. The solid lines represent the experimental work of efficiency at different power levels, measured by power analyzer WT333E and isolated sampling circuits. The accuracy of current and voltage measurement is about $0.1 \%$.

The peak efficiency of PFC is higher than $99 \%$ at $220 V_{\text {ac }}$ input. Under light load (about $10 \%$ of full load) conditions, the efficiency can still reach over $97 \%$. From the comparison between the calculation and experimental results of efficiency, the calculated results according to the loss model are close to the measured efficiencies with different load at $110 V_{\text {ac }}$ and 220 $V_{\text {ac }}$ respectively, which verifies the accuracy of the proposed uniform minimum loss model.

In order to validate the accuracy of the calculation model, thermal experiment was conducted at $1500 \mathrm{~W}$ and the result 


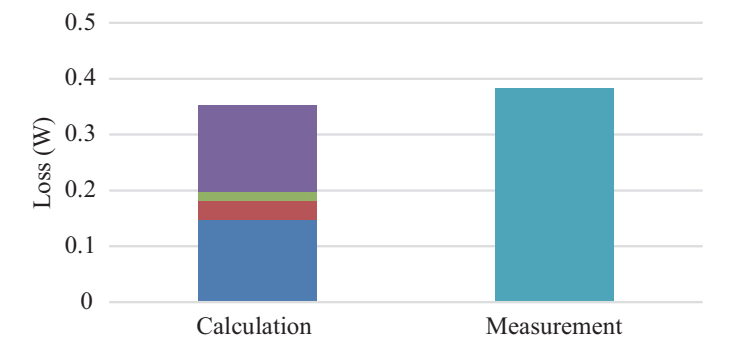

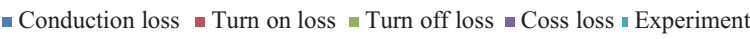

Fig. 22. Comparison between calculation and experiment.

is shown in Fig. 21. The ambient temperature is $26^{\circ} \mathrm{C}$ and the temperature rise is about $25^{\circ} \mathrm{C}$. Since the copper area we used is equivalent to the chip area $\left(10 \mathrm{~mm}^{2}\right)$, according to the literature [35], the thermal resistance is 1.45 times of that in the datasheet, which is $45^{\circ} \mathrm{C} / \mathrm{W}$. So, the loss of each device can be calculated as $0.38 \mathrm{~W}$. On the other hand, the theoretical calculation is $0.35 \mathrm{~W}$, whose loss breakdown is shown in Fig. 22. So, the calculation model matches the experimental result and only has a slight deviation of less than $10 \%$.

\section{SUMMARY AND CONCLUSIONS}

This paper proposed a uniform minimal loss model for $\mathrm{CHB}$ multilevel PFC converter, revealing that the minimal loss is only related to the NFoM of the individual device and has nothing to do with the device quantities. Based on the model, a design methodology of efficiency and density optimization for the multilevel PFC converter is proposed. According to the design methodology, 6-cell structure with $60 \mathrm{kHz}$ switching frequency is easily selected without complex calculation process. Finally, a prototype with $2 \mathrm{~kW}$ rated power is constructed and tested, achieving over $99 \%$ peak efficiency with power density over $1000 \mathrm{~W} / \mathrm{in}^{3}$.

\section{REFERENCES}

[1] IEA, "Global EV Outlook 2019," 2019.[Online].Available: https://www. iea.org/reports/global-ev-outlook-2019

[2] Z. Liu, B. Li, F. C. Lee, and Q. Li, "High-efficiency high-density critical mode rectifier/inverter for WBG-device-based on-board charger," in IEEE Transactions on Industrial Electronics, vol. 64, no. 11, pp. 91149123, Nov. 2017.

[3] National Resources Defense Council, "Data center efficiency assessment-Scaling up energy efficiency across the data center industry: Evaluating key drivers and barriers," 2014.[Online].Available:https:// www.nrdc.org/sites/default/files/data-center-efficiency-assessment-IP.pdf

[4] J. W. Kolar, "Design of a highly efficient $(97.7 \%)$ and very compact $(2.2$ $\mathrm{kW} / \mathrm{dm} 3$ ) isolated AC-DC telecom power supply module based on the multicell ISOP converter approach," in IEEE Transactions on Power Electronics, vol. 32, no. 10, pp. 7750-7769, Oct. 2017.

[5] Q. Huang and A. Q. Huang, "Review of GaN totem-pole bridgeless PFC," in CPSS Transactions on Power Electronics and Applications, vol. 2, no. 3, pp. 187-196, Sept. 2017.

[6] C. Zhao and X. Wu, "Accurate operating analysis of boundary mode totem-pole boost PFC converter considering the reverse recovery of mosfet," in IEEE Transactions on Power Electronics, vol. 33, no. 12, pp. 10038-10043, Dec. 2018

[7] J. E. Huber and J. W. Kolar, "Optimum number of cascaded cells for high-power medium-voltage AC-DC converters," in IEEE Journal of Emerging and Selected Topics in Power Electronics, vol. 5, no. 1, pp. 213-232, Mar. 2017.

[8] Z. Liu, F. C. Lee, Q. Li, and Y. Yang, "Design of GaN-based MHz totem-pole PFC rectifier," in IEEE Journal of Emerging and Selected Topics in Power Electronics, vol. 4, no. 3, pp. 799-807, Sept. 2016.

[9] D. Bortis, D. Neumayr, and J. W. Kolar, " $\eta \rho$-Pareto optimization and comparative evaluation of inverter concepts considered for the GOOGLE Little Box Challenge," in Proceedings of 2016 IEEE 17th Workshop on Control and Modeling for Power Electronics (COMPEL), Trondheim, 2016, pp. 1-5.

[10] Y. Lei, C. Barth, S. Qin, W.-C. Liu, I. Moon, A. Stillwell, D. Chou, T. Foulkes, Z. Ye, Z. Liao, and R. C. N. Pilawa-Podgurski, "A 2-kW single-phase seven-level flying capacitor multilevel inverter with an active energy buffer," in IEEE Transactions on Power Electronics, vol. 32, no. 11, pp. 8570-8581, Nov. 2017.

[11] W. G. Hurley and W. H. Wölfle, "Transformers and inductors for power electronics: theory, design and applications," New York: John Wiley \& Sons, 2013, pp. 55-91.

[12] S. Qin, Y. Lei, Z. Ye, D. Chou, and R. C. N. Pilawa-Podgurski, “A high-power-density power factor correction front end based on seven-level flying capacitor multilevel converter," in IEEE Journal of Emerging and Selected Topics in Power Electronics, vol. 7, no. 3, pp. 1883-1898, Sept. 2019.

[13] Q. Huang, Q. Ma, P. Liu, A. Q. Huang, and M. de Rooij, “3 kW four-level flying capacitor totem-pole bridgeless PFC rectifier with $200 \mathrm{~V} \mathrm{GaN}$ devices," in Proceedings of 2019 IEEE Energy Conversion Congress and Exposition (ECCE), Baltimore, MD, USA, 2019, pp. 81-88.

[14] B. J. Baliga, "Power semiconductor device figure of merit for high-frequency applications," in IEEE Electron Device Letters, pp. 455-457, 1989.

[15] D. Reusch, J. Strydom, and A. Lidow, "Improving system performance with eGaN $®$ FETs in DC-DC applications," in Proceedings of $2013 \mathrm{In}$ ternational Symposium on Microelectronics, 2013, pp. 764-769.

[16] D. Reusch, J. Strydom, and A. Lidow, "A new family of GaN transistors for highly efficient high frequency DC-DC converters," in Proceedings of 2015 IEEE Applied Power Electronics Conference and Exposition (APEC), Charlotte, NC, 2015, pp. 1979-1985.

[17] J. Wu and X. Wu, "FoM based optimal frequency and voltage level design for high efficiency high density multilevel PFC with GaN device," in Proceedings of 2020 IEEE Applied Power Electronics Conference and Exposition (APEC), New Orleans, LA, 2020, pp. 1911-1915.

[18] Il-Jung Kim, S. Matsumoto, T. Sakai, and T. Yachi, "New power device figure of merit for high-frequency applications," in Proceedings of International Symposium on Power Semiconductor Devices and IC's: ISPSD'95, 1995, pp. 309-314.

[19] X. Wu and H. Shi, "High efficiency high density 1 MHz 380-12 V DCX with low FoM devices," in IEEE Transactions on Industrial Electronics, vol. 67, no. 2, pp. 1648-1656, Feb. 2020.

[20] P. Qashqai, A. Sheikholeslami, H. Vahedi, and K. Al-Haddad, "A review on multilevel converter topologies for electric transportation applications," in Proceedings of 2015 IEEE Vehicle Power and Propulsion Conference (VPPC), Montreal, QC, 2015, pp. 1-6.

[21] A. Dekka, B. Wu, R. L. Fuentes, M. Perez, and N. R. Zargari, "Evolution of topologies, modeling, control schemes, and applications of modular multilevel converters," in IEEE Journal of Emerging and Selected Topics in Power Electronics, vol. 5, no. 4, pp. 1631-1656, Dec. 2017.

[22] T. Foulkes, T. Modeer, and R. C. N. Pilawa-Podgurski, "Developing a standardized method for measuring and quantifying dynamic on-state resistance via a survey of low voltage GaN HEMTs," in Proceedings of 2018 IEEE Applied Power Electronics Conference and Exposition (APEC), San Antonio, TX, 2018, pp. 2717-2724.

[23] “EPC2032 datasheet," 2020. [Online]. Available:https://epc-co.com/epc/ Portals/0/epc/documents/datasheets/epc2032_datasheet.pdf

[24] Vicor,"Isolated, Regulated DC Converter DCM3414x75H53C2yzz Datashee," 2021.[Online].Available:https://www.vicorpower.com/documents/datasheets/DCM3414x75H31C2yzz_ds.pdf

[25] M. H. Ahmed, A. Nabih, F. C. Lee, and Q. Li, "High-efficiency, high-density isolated/regulated $48 \mathrm{~V}$ bus converter with a novel planar magnetic structure," in Proceedings of 2019 IEEE Applied Power Elec- 
tronics Conference and Exposition (APEC), Anaheim, CA, USA, 2019, pp. 468-475.

[26] EPC appnote AN002,"Fundamentals of Gallium Nitride Power Transis tors," [Online].Available:https://epc-co.com/epc/Portals/0/epc/documents/product-training/Appnote_GaNfundamentals.pdf

[27] EPC appnote AN022,"Generation 5 eGaN Technology A Quantum Leap into a New Universe of Performance!'[Online].Available:https://epc-co. com/epc/Portals/0/epc/documents/application-notes/AN022\%20Generation $\% 205 \% 20 \mathrm{eGaN} \% 20$ Technology\%20EPC.pdf

[28] A. K. Mohammadd, "Gate capacitances of high electron mobility transistors," in Proceedings of International Confernece on Electrical \& Computer Engineering, Dhaka, Bangladesh, 2002, pp. 128-131.

[29] T. Fang, X. Ruan, and C. K. Tse, "Control strategy to achieve input and output voltage sharing for input-series-output-series-connected inverter systems," in IEEE Transactions on Power Electronics, vol. 25, no. 6, pp. 1585-1596, Jun. 2010.

[30] R. Giri, V. Choudhary, R. Ayyanar, and N. Mohan, "Common-duty-ratio control of input-series connected modular DC-DC converters with active input voltage and load-current sharing," in IEEE Transactions on Industry Applications, vol. 42, no. 4, pp. 1101-1111, Jul.-Aug. 2006.

[31] T. Zhao, G. Wang, S. Bhattacharya, and A. Q. Huang, "Voltage and power balance control for a cascaded H-bridge converter-based solid-state transformer," in IEEE Transactions on Power Electronics, vol. 28, no. 4, pp. 1523-1532, Apr. 2013.

[32] D. Sha, G. Xu, and Y. Xu, "Utility direct interfaced charger/discharger employing unified voltage balance control for cascaded H-bridge units and decentralized control for CF-DAB modules," in IEEE Transactions on Industrial Electronics, vol. 64, no. 10, pp. 7831-7841, Oct. 2017.

[33] E. Villanueva, P. Correa, J. Rodriguez, and M. Pacas, "Control of a single-phase cascaded H-bridge multilevel inverter for grid-connected photovoltaic systems," in IEEE Transactions on Industrial Electronics, vol. 56, no. 11, pp. 4399-4406, Nov. 2009.

[34] A. Dell'Aquila, M. Liserre, V. G. Monopoli, and P. Rotondo, "Overview of PI-based solutions for the control of dc buses of a single-phase H-bridge multilevel active rectifier," in IEEE Transactions on Industry Applications, vol. 44, no. 3, pp. 857-866, May-Jun. 2008.

[35] EPC appnote AN011, “Thermal Performance of eGaN FETs,"[Online]. Available:https://epc-co.com/epc/Portals/0/epc/documents/product-training/Appnote_Thermal_Performance_of_eGaN_FETs.pdf

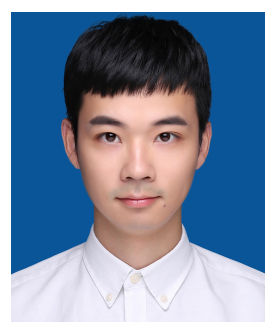

Jiawen Wu was born in Jiangsu Province, China. He received the B.S. degree from Beijing Institute of Technology, Peking, China, in 2017 and the M.S. degree from Zhejiang University, Hangzhou, China, in 2021. He is currently working with INOVANCE (Suzhou) Co., Ltd. His research interests include high density and high efficiency AC-DC and multilevel converters.

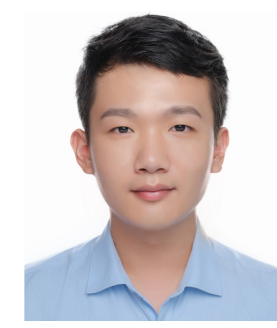

Yu Qi received the B.S. degree from Shanghai Jiao Tong University, Shanghai, China, in 2018, and the M.S. degree from Zhejiang University, Hangzhou, China, in 2021, both in electrical enginering.

$\mathrm{He}$ is currently working with Silergy Semiconductor Technology (Hangzhou) Co., Ltd., His research interests include high efficiency, high power density multilevel AC-DC converter and suppression methods of common-mode noise.

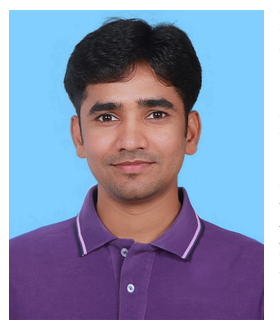

Faheem Muhammad received the Bachelor of Technology (Hons) degree in electrical technology from the University of Engineering and Technology Lahore, Pakistan, in 2015, and M.S. degree in electrical engineering from Xian Jiaotong University, Xian, China, in 2017. Since 2019, he has been perusing the $\mathrm{Ph} . \mathrm{D}$. degree with Zhejiang University, Hangzhou, China. From 2017 to 2019, he worked as an electrical product manager in CYG Sunri Co., Ltd., in Shenzhen, China. His research interest includes non-isolated DC/DC converter applications, high power density and high-efficiency DC/DC power converter applications, EMI control, OBC DC/DC power converters, and filters.

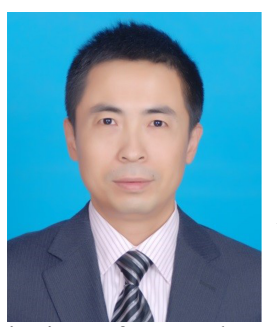

Xinke Wu received the B.S. and M.S. degrees in electrical engineering from Harbin Institute of Technology, Harbin, China, in 2000 and 2002, respectively, and received Ph.D. degree in electrical engineering from Zhejiang University, Hangzhou, China, in 2006. He was a postdoctoral fellow of National Engineering Research Center (NERC) for Applied Power Electronics in Zhejiang University from 2007 to 2009. Since 2009, he worked with institute of power electronics of Zhejiang University as an assistant research fellow from 2009 to 2010 then as an associate professor from 2011 to 2015 . And now, he serves as a professor. From 2011 to 2012, he was a visiting scholar in Center of Power Electronics System (CPES), Virginia Tech. He received Young Scholar award from Zhejiang University in 2012, Young Scholar Award from NSF of China in 2015 and Young Scholar Award from CPSS of China, 2017. Since 2016, he has been the Associate Editor of IEEE Transactions on Power Electronics. He received the First Prize of Best Transaction Paper Award from the IEEE Power Electronics Society in 2019.

His research covers high frequency, high power density and high efficiency power conversions, power electronics system integration. 